\title{
Functional roles of the E3 ubiquitin ligase HYD in Drosophila tissues
}

\author{
Iuliia Galimova \\ IMCB SB RAS, Novosibirsk, Russia \\ galimova@mcb.nsc.ru
}

\author{
Natalia Dorogova \\ ICG SB RAS, Novosibirsk, Russia \\ dorogova@bionet.nsc.ru
}

\author{
Svetlana Fedorova \\ ICG SB RAS, Novosibirsk, Russia \\ NSU, Novosibirsk, Russia \\ fsveta@bionet.nsc.ru
}

\begin{abstract}
Drosophila tumor suppressor HYD is required for the regulation of cell proliferation, growth and differentiation. HYD is involved in cell processes at different levels: it can regulate gene expression by binding to its promoters, it binds to some RNA for gene silencing and it participates in protein degradation due to its ubiquitin-ligase activity. Analysis of Drosophila hyd mutants revealed that HYD functions depend on tissue and stage of development: misexpression in larval somatic tissues causes overproliferation, defects of meiosis and spermatid differentiation in spermatogenesis, and massive cell death with occasional germline overproliferation during oogenesis.
\end{abstract}

Keywords - ubiquitin ligase, tumor suppressor, overproliferation, cell death, germline and somatic tissues

\section{Motivation and aim}

The Ubiquitin-Proteasome System (UPS) is an important regulator of cell signaling and proteostasis, which are essential to a variety of cellular processes. Components of UPS are implicated in regulation of cell cycle progression, signal transduction, DNA damage response, metabolism and transcriptional control. E3 ubiquitin ligases play key role in UPS functioning. Ubiquitin ligases (E3 enzymes) transfer ubiquitin from ubiquitin-conjugating (E2) enzymes to target proteins. By determing the selection of target proteins, modification sites on those target proteins, and the types of ubiquitin modifications that are formed, E3 enzymes are key specificity factors in ubiquitin signaling.

One of the most interesting E3 ligases is drosophila tumor suppressor HYD (hyperplastic disc). It is required for the regulation of cell proliferation during development. Mutations in the hyd gene result in developmental abnormalities that include adult sterility caused by germ cell defects [1]. In eye imaginal disc HYD function differs from what is expected for conventional tumor suppressor behaviour [2]. In eye disc homozygous hyd mutant clones induce non-autonomous overproliferation of nearby tissue [2].

UPS is highly conserved through evolution. Human orthologue of drosophila HYD - named EDD1 or UBR5 was identified in 1998 [3]. UBR5 expression is deregulated in many cancer types, amplification of UBR5 has been reported in human breast and ovarian cancer [4]. However, the mechanism by which UBR5 may contribute to tumor initiation and progression remains poorly defined.

\section{Methods}

To define the role of HYD in Drosophila germ line tissues and reveal the cause of sterility we studied the processes of spermatogenesis and oogenesis in drosophila hyd mutants. We used Bloomington Drosophila stocks: kni hyd15 e1 /TM3, Sb1 (3718) and yw; PBac $\{3 \mathrm{HPy}+\}$ hydC017/TM3,
Sb1 Ser1 (16256) as the source of mutant hyd alleles. Detailed cytological analysis of oogenesis and spermatogenesis was performed using fluorescent and electronic microscopy.

\section{Results and discussion}

Analysis of spermatogenesis showed that mutations of this gene cause multiple cell division abnormalities in spermatogenesis, but do not lead to hyperplasia of both generative and somatic tissue [5]. In contrast, main effect of hyd mutations in oogenesis was massive cell death. However, about $5 \%$ of ovaries contained egg chambers with abnormal amount of germ cells - 2-4 times higher than in control (32or 64-cell cysts in comparing to normal 16-cells). It indicates 1 or 2 extra rounds of cell division of mutant cystoblasts. It should be noted that hyd mutations did not affect somatic follicular cells.

Thus, in different tissues mutations of hyd lead to various consequences: overproliferation in somatic tissues, defects of meiosis and spermatid differentiation in drosophila spermatogenesis, and massive cell death with occasional overproliferation during oogenesis. So, HYD functions may differ depending on cell context. It was shown that HYD can regulate gene expression at transcriptional level [6] and translational level, owing to its unique feature - presence of PABC domain, commonly found in pABP proteins, whose function is related to mRNA translation. Also it is known that mammalian EDD plays a critical role in miRNA silencing [7] and participates in DNA damage response [8]. Interesting, that E3 ubiquitin ligase activity is dispensable for EDD function in miRNA silencing. The PABC domain of EDD is essential for its silencing function.

It is clear that HYD possess many apparently divergent roles in multiple pathways in different tissues. Earlier studies have shown that tumor suppressor genes can function in many tissues, but only in some of them they cause hyperplasia [9]. Futher researches are required to better define the role of HYD in different cell processes.

\section{ACKNOWLEDGMENT}

Supported by the ICG SB RAS budget Project (03242019-0042-C-01).

\section{REFERENCES}

[1] Mansfield E.A. et al. (1994). Genetic and molecular analysis of hyperplastic discs, a gene whose product is required for regulation of cell proliferation in Drosophila melanogaster imaginal discs and germ cells. Dev. Biol. 165(2): 507-526.

[2] Lee J.D. et al. (2002). The ubiquitin ligase Hyperplastic discs negatively regulates hedgehog and decapentaplegic expression by independent mechanisms. Development. 129(24): 5697-5706.

[3] Callaghan M.J. et al. (1998). Identification of a human HECT family protein with homology to the Drosophila tumor suppressor gene hyperplastic discs. Oncogene. 17: 3479-3491. 
[4] Clancy J.L. et al. (2003). EDD, the human orthologue of the hyperplastic discs tumour suppressor gene, is amplified and overexpressed in cancer. Oncogene. 22: 5070-5081.

[5] Pertceva J.A. et al. (2010) The role of Drosophila hyperplastic discs gene in spermatogenesis. Cell Biol. Int. 34(10): 991-996.

[6] Wang G. et al. (2014). Hyperplastic discs differentially regulates the transcriptional outputs of hedgehog signaling. Mech. Dev. 133: 117125.

[7] Su H. et al. (2011). Mammalian hyperplastic discs homolog EDD regulates microRNA-mediated gene silencing. Mol Cell. 43(1): $97-$ 109.
[8] Henderson M.J. et al. (2002). EDD, the human hyperplastic discs protein, has a role in progesterone receptor coactivation and potential involvement in DNA damage response. J. Biol. Chem.19;277(29):26468-78.

[9] Gateff E. (1994). Tumor suppressor and overgrowth suppressor genes of Drosophila melanogaster: developmental aspects. Int. J. Dev. Biol. 38(4):565-90. 